\title{
AXIALLY SYMMETRIC DYNAMICS OF PNe
}

\author{
LIFAN WANG \\ Dept. of Astronomy, The University of Manchester
}

and

Center for Astrophysics, The University of Science and Technology of China

Following Kahn \& West (1985), we investigate the formation of PNe in the slow and fast wind interaction scheme by assuming the slow wind axially symmetric. We have further assumed that the mass loss rate for the slow wind is not steady. It is found that the final morphology of the nebula depends not only upon the initial degrees of the seed asymmetry in the slow wind, but also upon the time variations of the mass loss rate. As an example, we show in some detail the case where the central star first blows an axially symmetric slow wind during its red giant stage, this wind is followed by a superwind while the star is on the AGB, these slow wind is later overtaken and shaped by the fast wind during the post-AGB branch. It is found that a small initial asymmetry can be amplified and reproduces the various observed morphologies of the PNe. 